\title{
Severe Defects in Dorsal Thalamic Development in Low- Density Lipoprotein Receptor-Related Protein-6 Mutants
}

\author{
Cheng-Ji Zhou, ${ }^{1}$ Kathleen I. Pinson, ${ }^{2}$ and Samuel J. Pleasure ${ }^{1}$ \\ ${ }^{1}$ Department of Neurology, Programs in Neuroscience and Developmental Biology, University of California, San Francisco, San Francisco, California 94143- \\ 0435, and 2Department of Reproductive Medicine, University of California, San Diego, La Jolla, California, 92093-0674
}

\begin{abstract}
Mice with mutations in the Wnt coreceptor low-density lipoprotein receptor-related protein-6 (LRP6) have a smaller and severely disorganized dorsal thalamus and lack thalamocortical projections. Using molecular markers, we showed that most dorsal thalamic and epithalamic neurons were missing, and most of the major dorsal thalamic nuclei were not identifiable. However, the ventral thalamus was essentially unaffected, although the dorsal thalamic defect leads to rostral displacement of portions of the ventral thalamus. Analysis of younger embryos showed that epithalamic and dorsal thalamic neurons were not produced at early stages of development, whereas ventral thalamic neurons were still produced. These defects were accompanied by improper formation of the boundary between dorsal and ventral thalamus, the zona limitans interthalamica (ZLI). Furthermore, the expression of an early marker of posterior forebrain development that marks the compartment from the midbrain-hindbrain junction to the ZLI (including the future dorsal thalamus, pretectum, and midbrain) was disrupted, supporting the idea that diencephalic development is abnormal from very early in embryogenesis. This study provides compelling in vivo evidence that thalamic development requires normal activity of the LRP6-mediated canonical Wnt signaling pathway.
\end{abstract}

Key words: diencephalon; differentiation; thalamus; Wnts; zona limitans; sonic hedgehog

\section{Introduction}

During vertebrate development, Wnts are expressed in highly localized domains in the dorsal neural tube and dorsomedial cerebral cortex, and strong evidence links Wnts to regulation of dorsal precursor proliferation in the spinal cord and medial cortical derivatives, like the hippocampus (Ikeya et al., 1997; Grove et al., 1998; Lee et al., 2000; Zhou et al., 2004). It is somewhat controversial as to whether Wnts also regulate cell fate in the developing mammalian nervous system, although this is well established in lower vertebrates (Dorsky et al., 1998). However, recent studies reexamining the phenotype of Wnt1/3A double mutants and gain-of-function experiments in chicks (Muroyama et al., 2002; Caspary and Anderson, 2003; Helms and Johnson, 2003) and examining the role of Wnts in mouse neural crest cell fate using conditionally mutant mice (Lee et al., 2004) have established that Wnts do appear to regulate cell fate in mammalian

\footnotetext{
Received June 1, 2004; revised July 19, 2004; accepted July 23, 2004.

This work was supported by grants from the National Institute of Neurological Disorders and Stroke, Burroughs Wellcome Fund, John Merck Fund, Whitehall Foundation, and National Alliance for Autism Research to S.J.P. We thank Bill Skarnes for allowing us to work on the LRP6 mutant mice; Grant Li and all other members of the Pleasure laboratory for helpful discussions and use of reagents; John Rubenstein for helpful discussions and numerous plasmids; Jeremy Nathans for SFRP2 plasmid; Andy McMahon for Wnt3, Wnt5a, and Wnt7b plasmids; Debra Bramblett and Ming-Jer Tsai for $\beta 4$ plasmid; Yasushi Nakagawa and Dennis 0'Leary for Ror- $\alpha$ plasmid; Hirohide Takebayashi for Olig2 antibody; Jhumku Kohtz for anti-DII antibody; V. A. Lee for RM0270; and the Developmental Studies Hybridoma Bank at the University of lowa (lowa City, IA) for Pax6 and Nkx2.2 antibodies.

Correspondence should be addressed to Dr. Samuel J. Pleasure, Department of Neurology, University of California, San Francisco, P.0. Box 0435, Room S-268, 513 Parnassus Avenue, San Francisco, CA 94143-0435. E-mail: samuelp@itsa.ucsf.edu.

DOI:10.1523/JNEUROSCI.2123-04.2004

Copyright $\odot 2004$ Society for Neuroscience $\quad$ 0270-6474/04/247632-08\$15.00/0
}

neural development. However, it is still unclear whether this is true in other regions of the mammalian CNS.

The thalamus is a crucial intermediate target for sensory input to the cortex and has a central role in regulating sensory processing. The prosomeric model of brain development divides the developing forebrain into neuromeric divisions called prosomeres arrayed in an anteroposterior (A-P) manner in the neuroaxis (Rubenstein et al., 1994). In this schema, the diencephalon (including the thalamus) arises from components of prosomeres p1 (the pretectum), p2 (the dorsal thalamus and epithalamus), p3 (the ventral thalamus), and hypothalamus. The boundary between dorsal and ventral thalamus is termed the zona limitans interthalamica (ZLI) and has been postulated to be a local signaling center (Larsen et al., 2001). The folding of the neural tube during gestation as the brain expands leads to the transition of these initial anteroposterior divisions into a dorsoventral orientation. Previous studies have shown that during submammalian vertebrate development, Wnt signaling is involved in the initial anteroposterior patterning of the neural tube and later in subdividing the brain into telencephalon, eye fields, and diencephalon (Heisenberg et al., 2001; Houart et al., 2002; Nordstrom et al., 2002). Also, an important functional role for Wnt signaling specifically in thalamic development has recently been suggested in chick, where Wnt signals were shown to be required at early stages to induce a posterior forebrain marker in the diencephalon and then again at later stages to induce dorsal thalamic markers within this posterior forebrain domain (Braun et al., 2003).

To study the function of the Wnt/ $\beta$-catenin pathway in the developing thalamus, we examined the phenotype of lowdensity lipoprotein receptor-related protein-6 (LRP6) mutant 

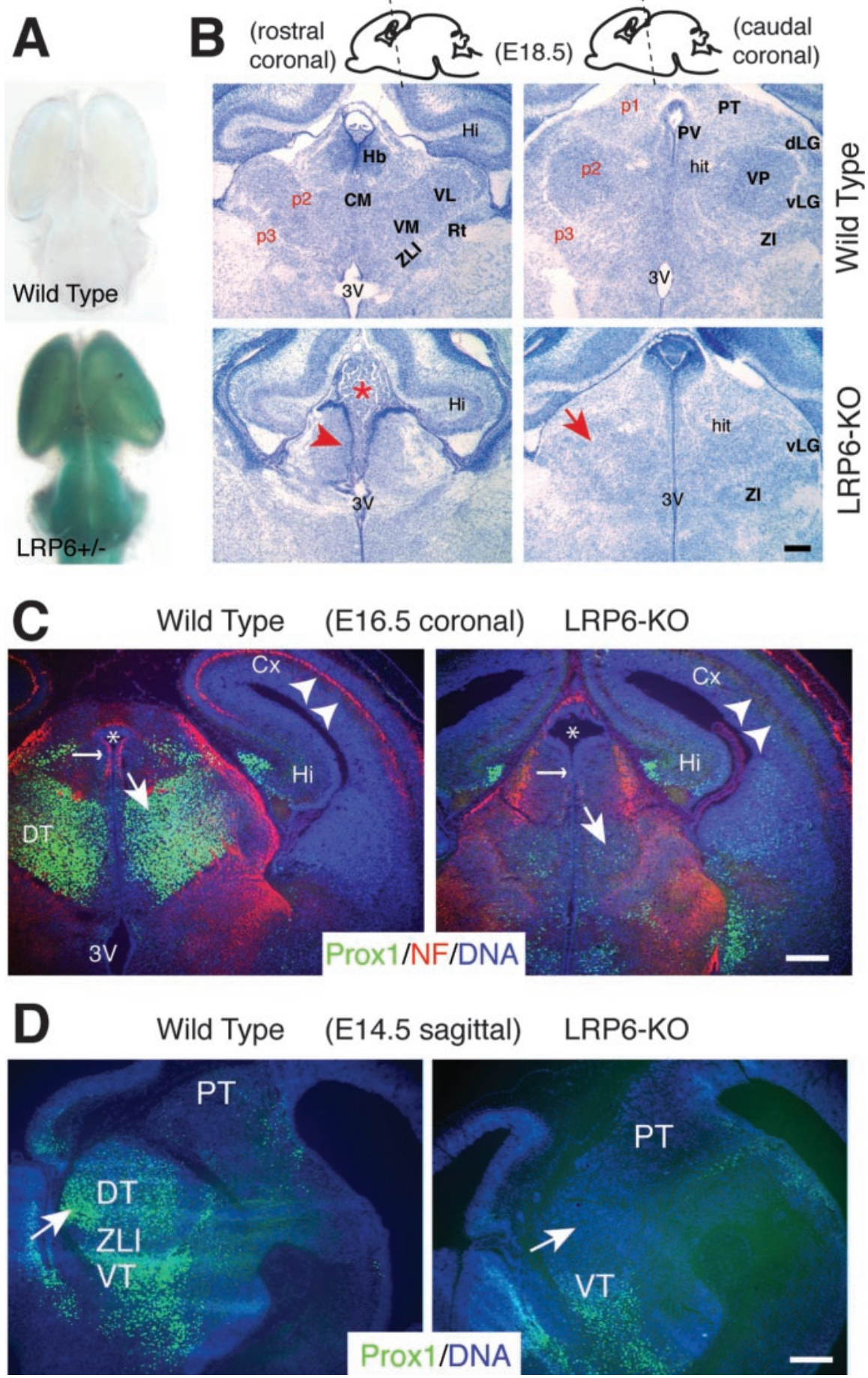

Figure 1. Disruption of the thalamus in LRP6 knock-out (K0) mice. $A, \mathrm{X}$-gal staining (blue) in heterozygous (LRP6+/-) shows that LRP6 is ubiquitously expressed in entire developing brain at E12.5. B, Nissl staining in E18.5 coronal brain sections shows anatomic defects of thalamus in mutants. These defects are most severe at rostral levels where all lateral thalamic structures are missing, and only the medial thalamic regions remained with a disorganized ventricular zone (red arrowhead). Note the extra choroid plexus-like structure in the third ventricle (red star). At caudal levels, the major posterior dorsal thalamic nuclei are not identifiable (red arrow). C, Double immunofluorescence for Prox1 (green; a dorsal thalamic neuronal marker) and neurofilament (red) in coronal sections at E16.5 shows that most dorsal thalamic neurons (large white arrows) are missing and that there are few axonal projections to the cortical subplate (where thalamocortical axons project prominently at this age) (white arrowheads) in the mutants. In addition, the habenular recess is larger in the mutant (asterisk), and the fibers normally coursing adjacent to the habenular ventricular zone (small white arrows) are missing or misplaced in the mutant. $D$, Immunofluorescence of Prox 1 (green) in sagittal brain sections of E14.5 mice shows a similar phenotype in the dorsal thalamus (white arrows). 3V, Third ventricle; $C \mathrm{X}_{\text {, }}$ cerebral cortex; CM, central medial thalamic nuclei; $\mathrm{dLG}$, dorsal lateral geniculate nucleus; DT, dorsal thalamus; Hb, habenular nuclei; Hi, hippocampal formation; hit, habenulo-interpenduncular tract; p1, 2, 3, prosomeres 1, 2, 3; PT, pretectum; PV, paraventricular thalamic nuclei; Rt, reticular thalamic nucleus; VL, ventral lateral thalamic nuclei; vLG, ventral lateral geniculate nucleus; VM, ventral medial thalamic nuclei; VP, ventral posterior thalamic nuclei; VT, ventral thalamus; ZI, zona incerta. Scale bars, $50 \mu \mathrm{m}$. mice. LRP6 is a ubiquitously expressed Wnt receptor that acts along with members of the Frizzled family to transduce Wnt signals through the Wnt/ $\beta$-catenin pathway (Pinson et al., 2000; Tamai et al., 2000; Wehrli et al., 2000). Thus, LRP6 mutants represent a generalized hypomorphic (because of complementation by LRP5) loss of function for the $\mathrm{Wnt} / \beta$-catenin pathway (Maretto et al., 2003; Kelly et al., 2004).

\section{Materials and Methods}

Animals and tissue preparation. All animals were treated according to protocols approved by the University of California, San Francisco. The LRP6 mutant mice were originally generated by gene trap insertion that joined the first 321 amino acids of the LRP6 protein in-frame with the $\beta$-geo reporter gene that is then trapped in the endoplasmic reticulum rendering it nonfunctional (Pinson et al., 2000). LRP6 mutant mouse embryos were obtained from timed matings. Females with semen plugs found the next morning were considered embryonic day 0.5 (E0.5). As described previously, the homozygous mutants showed severe anatomical defects in the limbs and tail and died at birth (Pinson et al., 2000). Embryos between E10.5 and E18.5 were genotyped by morphology and X-gal staining. Embryo heads or brains were fixed by immersion in $4 \%$ paraformaldehyde and sectioned on a cryostat at $7 \mu \mathrm{m}$. Two to five normal or mutant embryos were used for each experiment at each time point.

Antibodies and histology. Rabbit antibodies to prospero-related homeobox-1 (Proxl) (1:5000), and Histone H3 (1:2000; Upstate Biotechnology, Lake Placid, NY), and mouse antibodies to neurofilament (RMO270; 1:3000; gift from Virginia Lee, University of Pennsylvania, Philadelphia, PA), Nkx2.2 (1:1000), and Pax6 (1:1000) (Developmental Studies Hybridoma Bank, University of Iowa, Iowa City, IA) were used according to widely published immunohistologic protocols and detected with secondary antibodies conjugated to Alexa fluorochromes (Molecular Probes, Eugene, OR) followed by nuclei counterstaining with Hoechst 33258 (Sigma, St. Louis, MO).

For bromodeoxyuridine (BrdU) labeling, pregnant mice were injected intraperitoneally with $100 \mathrm{mg} / \mathrm{kg}$ body weight and then the embryo brains were dissected out $1 \mathrm{hr}$ later. The cryostat sections were pretreated with $2 \mathrm{~N} \mathrm{HCl}$ for $30 \mathrm{~min}$ at $37^{\circ} \mathrm{C}$ followed by general immunofluorescence with anti-BrdU antibody (1:1000; Roche Products, Hertforshire, UK). For X-gal staining, embryo brains were dissected into $2 \%$ paraformaldehyde for $1 \mathrm{hr}$ and stained with X-gal substrate solution.

Nonradioactive in situ hybridization histochemistry was performed using digoxigeninlabeled riboprobes for $\beta 4, \mathrm{Dlx} 2, \mathrm{Gbx} 2$, Id $4, \mathrm{Ne}-$ trin, retinoic acid receptor-related orphan receptor $(\operatorname{ROR} \alpha)$, soluble Frizzled-related protein-2 (SFRp2), Shh, Tcf4, Irx3, Six3, Wnt3, 


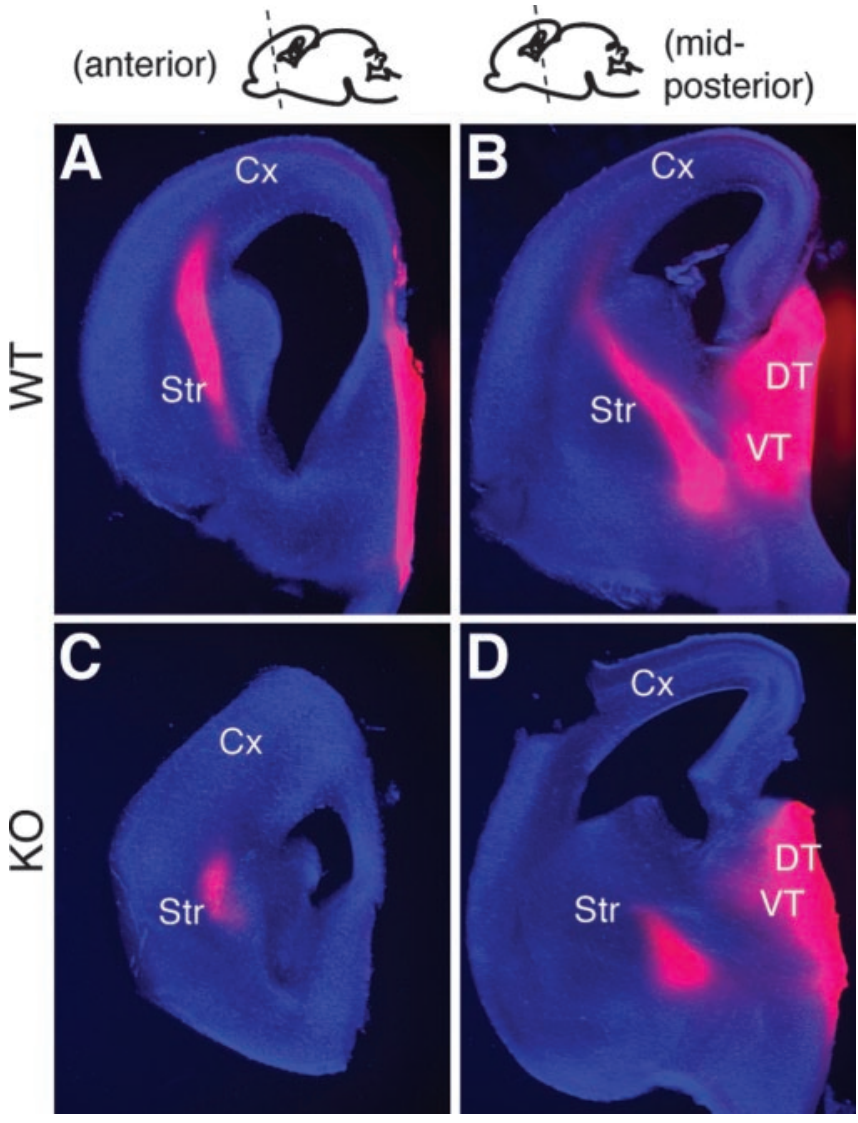

Figure 2. LRP6 mutants fail to produce thalamocortical projections. $A-D$, E18.5 wild-type $(A, B)$ and mutant $(C, D)$ brains after placement of Dil crystal in the thalamus. Wild-type mice, shown at two coronal levels, have fibers from the dorsal thalamus projecting through the internal capsule to the cortex dorsally. In mutants, the fibers that leave the thalamus get stalled and terminate in the striatum. Str, Striatum; CX, cortex; DT, dorsal thalamus.

Wnt5a, and Wnt7b and detected with sheep anti-digoxigenin Fab fragments conjugated to alkaline phosphatase and then color developed with the enzyme substrate solution as described previously (Kim et al., 2001).

Lipophilic dye labeling was performed in fixed brains. Brains were hemisected in the sagittal plane and large crystals of DiI placed in the thalamus using fine forceps. Brains were stored in $4 \%$ paraformaldehyde (changed weekly) at $37^{\circ} \mathrm{C}$ in the dark for $6-8$ weeks. Brains were then sectioned and photographed.

\section{Results}

\section{LRP6 mutants have thalamic defects}

LRP6 was ubiquitously expressed during development of the brain (Fig. $1 A$ ), as shown previously in other embryonic regions (Pinson et al., 2000; Kelly et al., 2004), and LRP6 mutant mice had distinctive thalamic abnormalities evident by midgestation (Fig. 1). In Nissl stained sections, the dorsal thalamic compartment was affected, and it was difficult to identify individual dorsal thalamic nuclei, particularly more rostrally (Fig. $1 B$ ). This was also noticeable when we stained sections with antiserum for the divergent homeobox gene Prox1, which is expressed strongly in most dorsal thalamic neurons (Bagri et al., 2002). In these sections, very few Prox1-immunolabeled dorsal thalamic neurons were detected compared with wild-type mice in both coronal and sagittal section planes (Fig. 1C,D). In addition, the third ventricle and habenular recess were enlarged, and there was excess choroid plexus in the third ventricle (Fig. $1 B, C$ ). In parasagittal sections, the anteroposterior boundaries between the pretectum-dorsal thalamus and dorsal-ventral thalamus were less distinct, and the

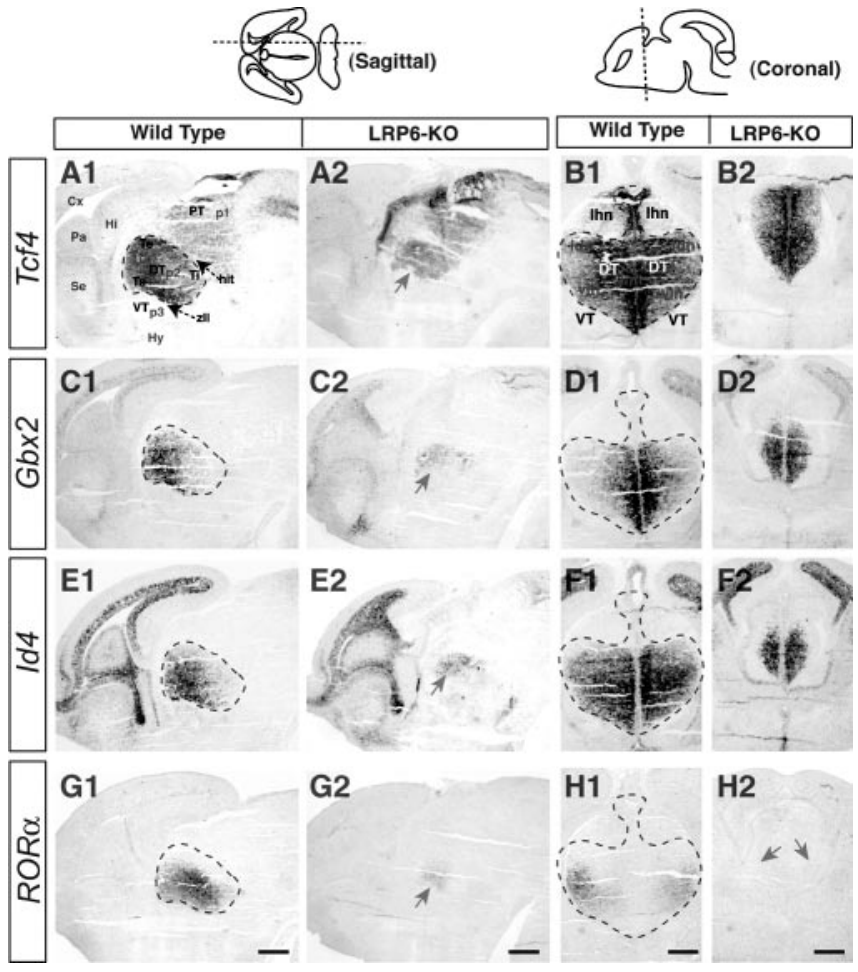

Figure 3. Molecular anatomy of the dorsal thalamus in LRP6 mutants. $A-H$, In situ hybridization of dorsal thalamic markers $\operatorname{Tcf} 4(A, B), \mathrm{Gbx2}(C, D), \operatorname{Id} 4(E, F)$, and $\operatorname{ROR} \alpha(G, H)$ are used to examine the details of the thalamic defects in sagittal and coronal sections at middle-late gestation. The black dashed line area $(A-H)$ indicates the entire dorsal thalamic region as defined by the expression of Tcf4 in adjacent sections. The arrows $(A 2, C 2, E 2, G 2, H 2)$ are used to show the location of residual staining for each marker in the small remaining dorsal thalamic structure. In LRP6 knock-out (KO) mice, the anterior, ventral, and lateral regions of the dorsal thalamus are most affected. ROR $\alpha$ is a marker for thalamic sensory relay neurons $(25)(G, H)$, and these neurons are especially severely affected in the mutant brains. C $x$, Cerebral cortex; DT, dorsal thalamus; Hi, hippocampus; hit, habenulo-interpenduncular tract; Hy, hypothalamus; imt, intermediate thalamic neuroepithelium; Idn, laterodorsal nuclei; Ihn, lateral habenular nucleus; $\mathrm{p1}$, 2, 3, prosomere 1, 2, 3; Pa, pallidal ganglion; $\mathrm{PT}$, pretectum; $\mathrm{SE}$, septal ganglion; $\mathrm{Ta}$, anterior thalamic region; Te, epithalamus; Ti, internal thalamic region; vln, ventral lateral nuclei; VT, ventral thalamus. Scale bars, $100 \mu \mathrm{m}$.

reduced size and disorganization of the dorsal thalamus were quite apparent (Fig. 1D).

\section{LRP6 mutants lack thalamocortical projections}

We noticed decreased numbers of neurofilament-stained axonal fibers in the subcortical white matter of mutant brains (Fig. 1C, arrowheads), implying that, in addition to its apparent anatomic disorganization, the dorsal thalamus may fail to appropriately produce thalamocortical projections. DiI tracing in E18.5 wildtype brains with dye crystals placed into the thalamus strongly labeled fibers leaving the thalamus and projecting laterally into the internal capsule before heading dorsally to penetrate the subcortical white matter (Fig. $2 A, B$ ). In mutant brains, some DiIlabeled fibers still projected out of the thalamus but failed to reach the cortex (Fig. 2C,D). These findings suggest that even those dorsal thalamic neurons that might remain in the mutants may be functionally abnormal and unable to produce axons that properly pathfind to the cortex.

We have shown previously that the telencephalon of LRP6 mutant mice is somewhat abnormal (Zhou et al., 2004); however, the most obvious defect (in the dentate gyrus) has no bearing on the ability of thalamocortical axons to pathfind to the cortex. We 


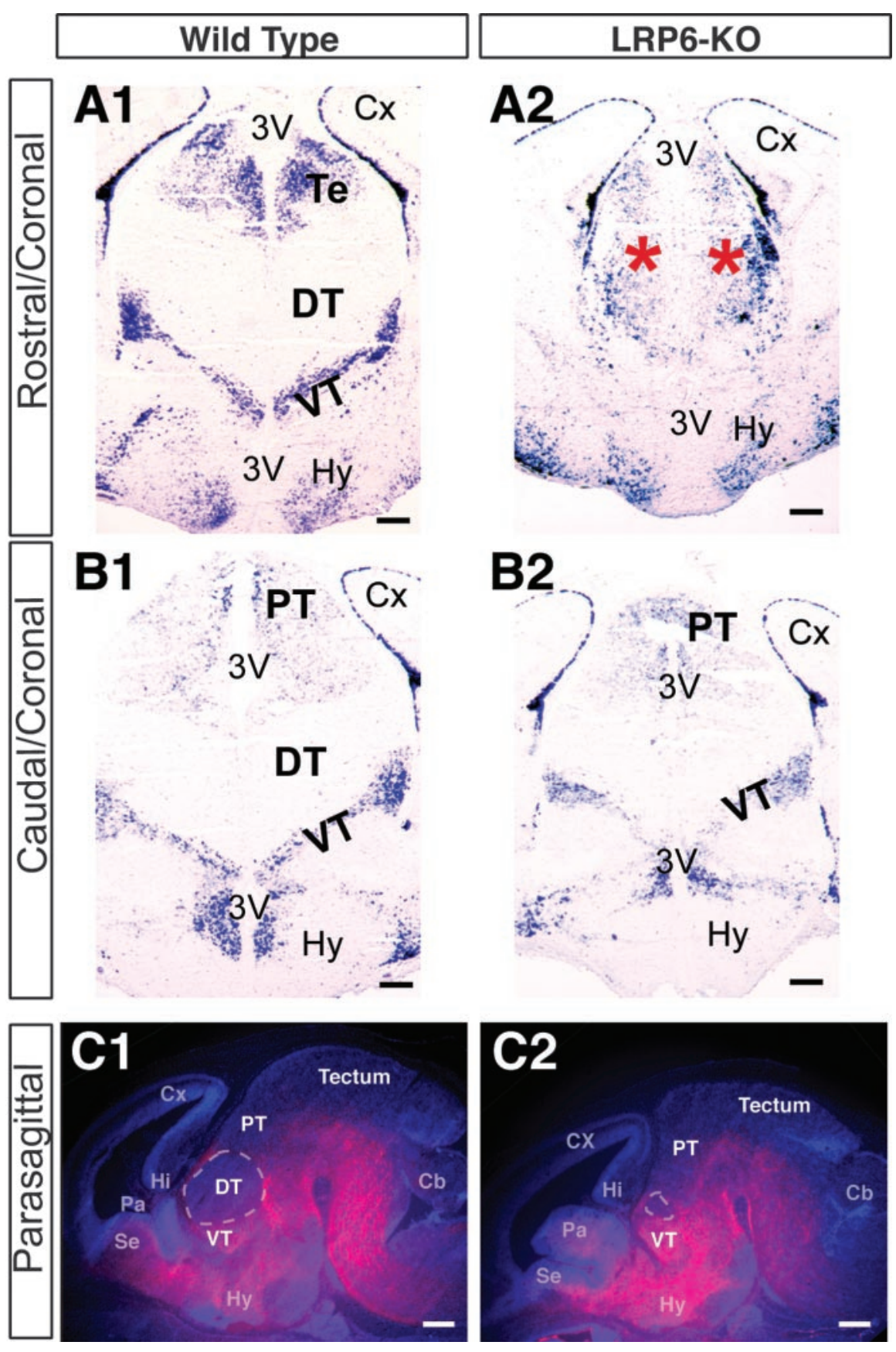

Figure 4. Analysis of ventral thalamic markers in middle-late gestation. $A 1-B 2$, Reelin expression in coronal brain sections (E15.5) by in situ hybridization shows that the caudal ventral thalamus appears quite normal $(B 1, B 2)$, whereas the rostral ventral thalamus may have been partially displaced by the absence of the dorsal thalamus rostrally ( $A 2$, red stars). Note that the reelin expression pattern in other regions, including pretectum and hypothalamus of the wild type and mutants, is also comparable. C1, C2, Immunofluorescence of GAD65 (red) in E17.5 sagittal brain sections (with a DNA staining in blue) shows that the GAD65-immunopositive ventral thalamus is largely intact, whereas the rostral portion of the ventral thalamus is shifted dorsally in the mutants. This is consistent with the reelin expression in the mutant rostral ventral thalamus (A2). Note that the anatomical structure of the tectum (dorsal midbrain) appears normal in the mutants. The white dashed line area indicates the normal and defective dorsal thalamus $(C 1, C 2)$. 3V, Third ventricle; $C b$, cerebellum; CX, cerebral cortex; DT, dorsal thalamus; Hi, hippocampus; Hy, hypothalamus; Pa, pallidal ganglion; PT, pretectum; SE, septal ganglion; Te, epithalamus; VT, ventral thalamus. Scale bars, $100 \mu \mathrm{m}$.

have recently noted additional neocortical abnormalities in LRP6 mutants, but these also are unlikely to be germane to this question because these defects are primarily attributable to defects in precursor proliferative behavior in the cortical wall (Zhou et al., 2004).
Loss of multiple dorsal thalamic molecular markers in mutant mice The histologic abnormalities and loss of one marker (Prox1) in the dorsal thalamus of mutant mice suggested that there were alterations in the proper production of differentiated dorsal thalamic neurons. To evaluate this more fully, we examined the expression of several other more established dorsal thalamic neuronal markers, Tcf4, Gbx2, and Id4 (Miyashita-Lin et al., 1999; Rubenstein et al., 1999), in coronal and sagittal brain sections of late gestation embryos (Fig. $3 A-H)$. Tcf4 was broadly expressed in the pretectum, epithalamus, and dorsal thalamus of wild-type mice, whereas in mutants, the dorsal thalamic component of the Tcf expression domain was reduced (Fig. $3 A, B$ ). Gbx2 and Id 4 were both more limited to the dorsal thalamus in wild-type mice but were also dramatically reduced in their expression in the dorsal thalamus of mutant mice (Fig. $3 C-F)$. Within the dorsal thalamus, the most affected subregions were the anterior and ventrolateral regions, the site of important primary sensory neurons. Because thalamic primary sensory neurons express retinoic acid receptor-related orphan receptor $\operatorname{ROR} \alpha$ (Steinmayr et al., 1998; Nakagawa and O'Leary, 2002), we examined the distribution of this marker and found that LRP6 mutants almost entirely lacked expression of $\operatorname{ROR} \alpha$ (Fig. 3G,H).

These results raise an interesting question. Why were Proxl and $\operatorname{ROR} \alpha$ almost entirely missing in the mutant brains, whereas the other thalamic markers were still expressed in a medial domain of the dorsal thalamus? Because Prox1 was expressed only relatively late in thalamic development (not easily detectable with our antibody until E13.5; data not shown) and $\operatorname{ROR} \alpha$ is expressed in only a restricted subset of neurons, it seems likely that these two are expressed in a more differentiated state for dorsal thalamic neurons, whereas Id4, Gbx2, and Tcf4 are expressed considerably earlier. Thus, we conclude that LRP6 mutants had developmental disorganization of the dorsal thalamus, failure to properly project thalamocortical fibers to the cortex, and reduced expression of five separate dorsal thalamic molecular markers.

Formation of the ventral thalamus in LRP6 mutants

We also examined two ventral thalamic neuronal markers, Reelin and glutamate decarboxylase (GAD), to determine whether the diencephalic defects include the ventral thalamus. In contrast to 
the situation in the dorsal thalamus, the ventral thalamus appeared largely intact in the mutant mice (Fig. 4A-C). Interestingly, in the rostralmost diencephalon, in the most abnormally formed region of the dorsal thalamus, there was displaced expression of Reelin (Fig. 4A). This may be attributable to failure to suppress ventral thalamic markers from the dorsal thalamic territory as suggested recently in chick (Braun et al., 2003). Alternatively, this could also be caused by simple displacement of the ventral thalamus rostrally or ectopic migration of ventral thalamic neurons.

Early neuronal defects in LRP6 mutants To determine whether dorsal thalamic development is deranged in LRP6 mutants caused by early defects in neuron production rather than a secondary destructive lesion, we examined the expression of markers for the dorsal thalamus, epithalamus, ventral thalamus, and the compartmental boundary between them, the ZLI at earlier ages. In coronal brain sections of early embryos (E12), the epithalamic neuronal marker $\beta 4$ (Bramblett et al., 2002) was normally expressed selectively in epithalamic neurons, whereas in mutants, these cells were missing (Fig. 5). In wildtype mice, Gbx2 was abundantly expressed in paired stripes of neurons in the dorsal thalamus adjacent to the ventricular zone and were missing at this coronal level in the mutant diencephalon (Fig. 5). The axon guidance molecule Netrin and the transcription factor Nkx2.2 were expressed at the ZLI in wildtype mice but not in the mutants (Fig. 5) (data not shown). Dlx2 mRNA and protein expression, early markers of ventral thalamic neurons, occupied the area vacated by the dorsal thalamic markers in the mutant embryos (Fig. 5). We found no evidence of increased cell death at any age in the diencephalon of mutant mice (data not shown). Therefore, it seems that in LRP6 mutants, dorsal thalamic and epithalamic neurons were not properly generated and that the ZLI was also not properly formed. The expression of Dlx $2+$ cells more dorsally could be attributable to ectopic production of these cells or abnormal migration because of a breakdown of the normal boundary function of the ZLI (Larsen et al., 2001), perhaps even related to the loss of Netrin expression, which can act as a chemorepellant for migrating neurons (BlochGallego et al., 1999).

\section{Defects in thalamic neural precursor domains, Wnt ligands, and ZLI signaling molecule function}

To determine whether the failure to produce certain types of thalamic neurons was associated with alterations in the distribution of regionally specified neuronal precursor zones, we examined markers that distinguish these precursor domains. The Wnt responsive transcription factor Tcf 4 and the Wnt ligand Wnt3 were expressed in the ventricular zone of the epithalamus and dorsal thalamus in wild-type mice at this stage, but in mutants, the level of expression and size of the domain of expression were dramatically reduced (Fig. 6). In wild-type mice, the boundary between the dorsal and ventral thalamus (the ZLI) expressed Shh and Wnt5a, but in LRP6 mutants, the expression of both was missing at E12 in the coronal plane (Fig. 6). In marked contrast, expression of the ventral thalamic ventricular zone markers SFRP2 (a Wnt inhibitor) and Wnt7b (a Wnt ligand) was largely intact (Fig. 6). Because our analysis showed that the dorsal thalamic compartment and the segmental boundary between dorsal and ventral thalamus were abnormal, even at the point when precursor domains were established, it seemed likely that the phenotype begins even earlier in gestation at the time that diencephalic patterning is established. This was supported by our failure to find any obvious alteration in the distribution of dividing cells in the diencephalic ventricular zone between E10.5 and E13.5, indicating that the failure to produce an organized dorsal thalamus was not attributable to wholesale failure of precursor proliferation (data not shown).

\section{Early defects in diencephalic development in LRP6 mutants}

It was quite apparent from our analysis that the relationship of the ZLI and its expression of Shh to the loss of dorsal and epithalamic markers was likely to be important. Previous studies showed that the ZLI initially forms as a discrete compartment in the chick brain that subsequently is restricted to forming the thin boundary domain between prosomeres 2 and 3 (Larsen et al., 2001; Zeltser et al., 2001). We examined wild-type and LRP6 mutant embryos to determine whether ZLI defects were apparent at these earlier stages. Lunatic fringe was reported to be a marker for this domain at early stages of chick development (Zeltser et al., 2001), but we were unable to detect lunatic fringe in this region of the mouse brain (data not shown). By approximately E9.5, the 


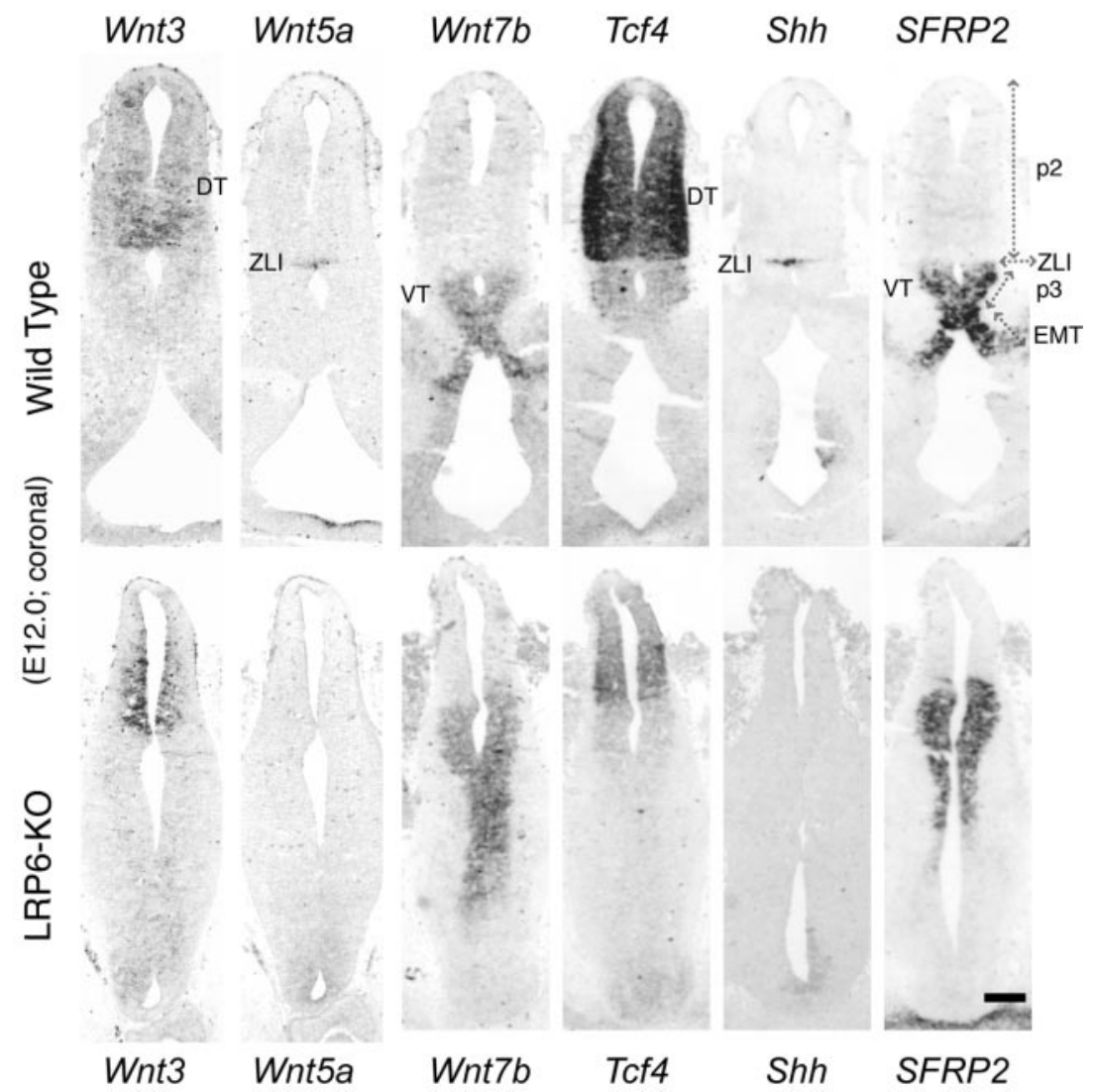

Figure 6. Progenitor zones and compartmentalization of the thalamus. Tcf4 and Wnt3 mark the ventricular zone of prosomere 2 (p2), including DT and Te, Shh and Wnt5a mark the p2/p3 boundary, and SFRP2 and Wnt7b mark the ventricular zone of p3 (VT) and eminentia thalami (EMT). In LRP6 knock-out (KO) mutant brains, only weak expression of Tcf4 and Wnt3 is seen and no expression of Shh or Wnt5a. However, the ventral thalamic ventricular zone markers are intact. It is difficult to be sure whether the ventral thalamic ventricular zone is truly expanded or somewhat displaced rostrally because of the smaller size of the rostral dorsal thalamus. Scale bars, $50 \mu \mathrm{m}$.

Shh expression spreads from the floor plate in a spur of expression to form the ZLI expression domain (Ishibashi and McMahon, 2002), and we were easily able to confirm this spur of expression in wild-type embryo brains by whole-mount in situ hybridization (Fig. 7A). Between E10.5 and E11.5, Gbx2 expression also became well established in the forming dorsal thalamus (Fig. $7 \mathrm{~B}, \mathrm{C}$ ). In contrast, in mutant embryos, Shh expression did not fully extend from the floor plate dorsally, and Gbx2 was missing entirely at these ages (Fig. $7 A-C$ ).

There are no reliable specific markers for the future dorsal thalamus before the formation of the ZLI. However, recently in chick, Irx3 was shown to be a marker for the forebrain posterior to the ZLI and Six3 a marker anterior to the ZLI from early in gestation (Braun et al., 2003). We were able to confirm that Irx3 was expressed in the future dorsal thalamus and pretectum (prosomeres P1 and P2) (Fig. 7C) but could not confirm the expression of Six 3 in the future ventral thalamus, rather, it was expressed in the hypothalamus and eye fields (data not shown). In LRP6 mutants, the Irx3 expression domain was considerably decreased in distribution (Fig. 7C). This supports the idea that dorsal thalamic development was defective from the earliest developmental stages.

\section{Discussion}

Our analysis with a variety of markers shows that the formation of the dorsal thalamus is disrupted in LRP6 mutant mice. This de- fect is first apparent very early in the development of the diencephalon, with early defects in the expression of a posterior forebrain marker and a failure to properly form the boundary between dorsal and ventral thalamus.

\section{Defects in ZLI signaling molecule expression in LRP6 mutants}

The failure of Shh expression in the ZLI in LRP6 mutant mice to properly extend dorsally and the abnormalities in two Shh pathway responsive genes $(\mathrm{Nkx} 2.2$ and Netrin) provide strong evidence that Shh signaling is defective locally in the diencephalon. We also found that Wnt5a, a noncanonical Wnt ligand, expression is missing at the ZLI. Thus, normal Wnt signaling early in diencephalic development is required for the production of this secondary signaling structure. Whether the loss of dorsal thalamic neurons is a reflection of a cell autonomous loss of Wnt signaling in the dorsal thalamus or attributable to a secondary loss of Shh signaling is currently unclear. However, the even earlier alterations in Irx3 expression and the previous studies in chick imply that there is a direct requirement for Wnt signaling (Braun et al., 2003).

Interestingly, a recent study showed that loss of Shh signaling in Shh null mice also leads to diencephalic defects. These mice lack Shh protein in the floorplate from early development and in the ZLI later and also have reduced Tcf4 expression in the thalamus (Ishibashi and McMahon, 2002). Also, a recent analysis of the role of Shh signaling in thalamic development in chicks showed that Shh directly induces dorsal thalamic markers in tissue explants (Hashimoto-Torii et al., 2003). These studies, together with the work described here and the recent analysis of the direct role of Wnt ligands in diencephalic development (Braun et al., 2003), imply that integrity of both pathways is crucial. Whether the Wnt and Shh pathways directly collaborate molecularly or work separately to induce dorsal thalamic development remains to be examined.

\section{Is there a reiterative role of Wnts in anteroposterior patterning in the brain?}

Recent studies in zebrafish showed that a gradient of Wnt signaling regulates the anteroposterior partitioning of the brain with high Wnt tone inducing diencephalic fate at the expense of more anterior structures, whereas inhibition of high Wnt tone is required to induce the formation of the telencephalon and eyes (Masai et al., 1997; Heisenberg et al., 2001; Houart et al., 2002). Other studies showed regulation of anteroposterior neural fate in the neural tube as a whole in chick by Wnts (Nordstrom et al., 2002; Gunhaga et al., 2003). Taken with this earlier work, the recent study showing that Wnts are also required to properly induce posterior patterning of the diencephalon in chick supports a repeated role for Wnt signaling in regulating A-P development of several forebrain compartments during development. 
Our analysis is quite consistent with these studies in that we found loss of dorsalposterior thalamus and the boundary between anterior and posterior thalamus in the diencephalon in the setting of decreased Wnt signaling tone. The importance of the LRP6 mutants is that they extend this phenomenon to mammals and establish that Wnt signaling is required. It is likely that LRP6 mutants are so useful only because of the redundancy with LRP5; however, it remains to be seen whether these receptors are functionally identical in signaling activity but differ only subtly in expression levels and timing. A recent study examining this issue also showed a role for LRP6 and LRP5 in A-P patterning in the early embryo and showed that compound mutants of these two receptors are far more severely affected and at earlier stages (Kelly et al., 2004). Thus, it seems likely that Wnt signaling regulates anteroposterior development of the nervous system in a reiterated manner at multiple stages of embryonic development to allow proper axis formation within each successively more restricted brain compartment. This would make the role of Wnt signaling in brain development functionally quite similar to neural crest development, where recent work showed reiterative roles for Wnt signaling over several developmental stages (Lewis et al., 2004).

\section{References}

Bagri A, Gurney T, He X, Zou YR, Littman DR, Tessier-Lavigne M, Pleasure SJ (2002) The chemokine SDF1 regulates migration of dentate granule cells. Development 129:4249-4260.

Bloch-Gallego E, Ezan F, Tessier-Lavigne M, Sotelo C (1999) Floor plate and netrin- 1 are involved in the migration and survival of inferior olivary neurons. J Neurosci 19:4407-4420.

Bramblett DE, Copeland NG, Jenkins NA, Tsai MJ (2002) BHLHB4 is a bHLH transcriptional regulator in pancreas and brain that marks the dimesencephalic boundary. Genomics 79:402-412.

Braun MM, Etheridge A, Bernard A, Robertson CP, Roelink H (2003) Wnt signaling is required at distinct stages of development for the induction of the posterior forebrain. Development 130:5579-5587.

Caspary T, Anderson KV (2003) Patterning cell types in the dorsal spinal cord: what the mouse mutants say. Nat Rev Neurosci 4:289-297.

Dorsky RI, Moon RT, Raible DW (1998) Control of neural crest cell fate by the Wnt signalling pathway. Nature 396:370-373.

Grove EA, Tole S, Limon J, Yip L, Ragsdale CW (1998) The hem of the embryonic cerebral cortex is defined by the expression of multiple Wnt genes and is compromised in Gli3-deficient mice. Development 125:2315-2325.

Gunhaga L, Marklund M, Sjodal M, Hsieh JC, Jessell TM, Edlund T (2003) Specification of dorsal telencephalic character by sequential Wnt and FGF signaling. Nat Neurosci 6:701-707.

Hashimoto-Torii K, Motoyama J, Hui CC, Kuroiwa A, Nakafuku M, Shimamura K (2003) Differential activities of Sonic hedgehog mediated by Gli transcription factors define distinct neuronal subtypes in the dorsal thalamus. Mech Dev 120:1097-1111.

Heisenberg CP, Houart C, Take-Uchi M, Rauch GJ, Young N, Coutinho P, Masai I, Caneparo L, Concha ML, Geisler R, Dale TC, Wilson SW, Stemple DL (2001) A mutation in the Gsk3-binding domain of zebrafish
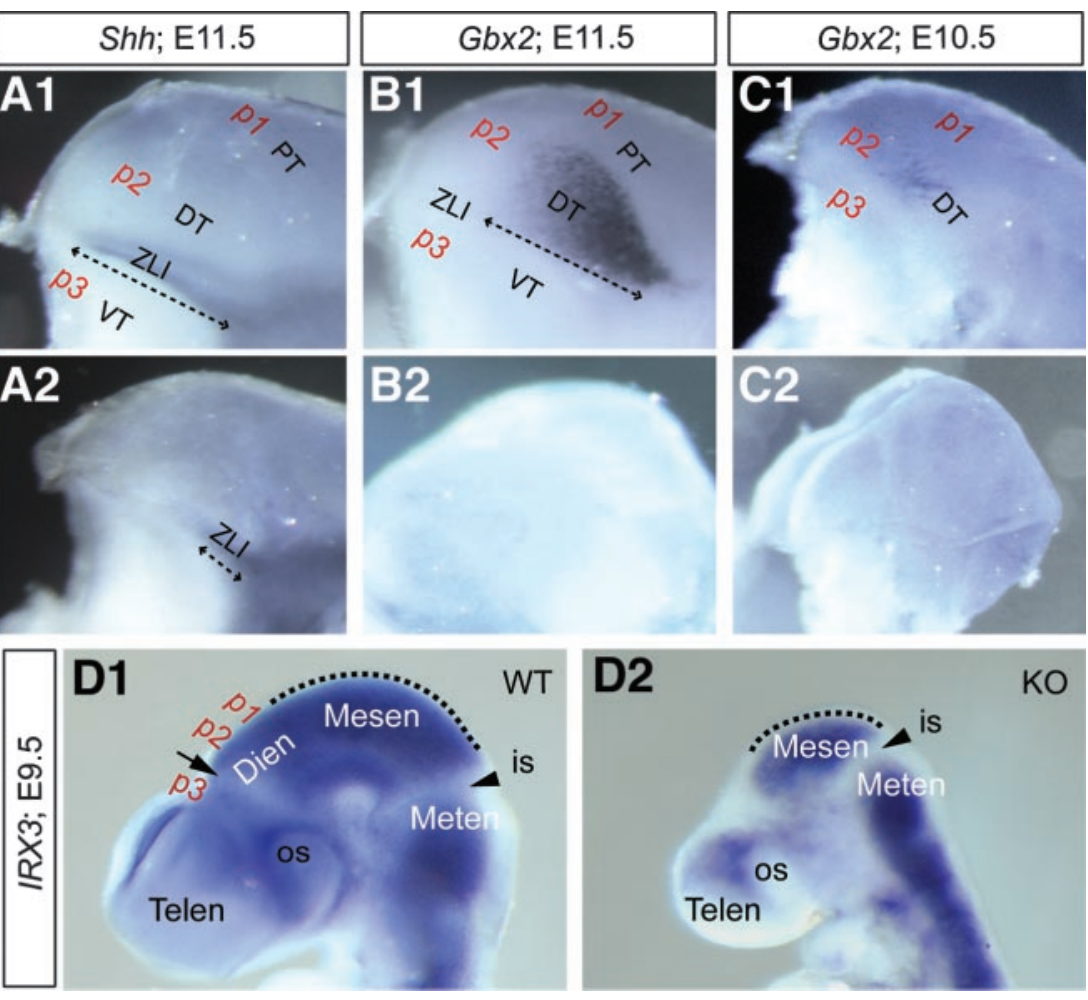

Figure 7. Early diencephalic defects in LRP6 mutants. A1-C2, Whole-mount in situ hybridization for Shh and Gbx2 in E10.5 and 列 l thalamus; is, isthmus; Mesen, mesencephalon; Meten, metencephalon; 0s, optic stalk; p1, 2, 3, prosomere 1, 2, 3; PT, pretectum; Telen, telencephalon; VT, ventral thalamus.

Masterblind/Axin 1 leads to a fate transformation of telencephalon and eyes to diencephalon. Genes Dev 15:1427-1434.

Helms AW, Johnson JE (2003) Specification of dorsal spinal cord interneurons. Curr Opin Neurobiol 13:42-49.

Houart C, Caneparo L, Heisenberg C, Barth K, Take-Uchi M, Wilson S (2002) Establishment of the telencephalon during gastrulation by local antagonism of Wnt signaling. Neuron 35:255-265.

Ikeya M, Lee SM, Johnson JE, McMahon AP, Takada S (1997) Wnt signalling required for expansion of neural crest and CNS progenitors. Nature 389:966-970.

Ishibashi M, McMahon AP (2002) A sonic hedgehog-dependent signaling relay regulates growth of diencephalic and mesencephalic primordia in the early mouse embryo. Development 129:4807-4819.

Kelly OG, Pinson KI, Skarnes WC (2004) The Wnt co-receptors Lrp5 and Lrp6 are essential for gastrulation in mice. Development 131:2803-2815.

Kim AS, Anderson SA, Rubenstein JL, Lowenstein DH, Pleasure SJ (2001) Pax- 6 regulates expression of SFRP- 2 and Wnt-7b in the developing CNS. J Neurosci 21:RC132.

Larsen CW, Zeltser LM, Lumsden A (2001) Boundary formation and compartition in the avian diencephalon. J Neurosci 21:4699-4711.

Lee HY, Kleber M, Hari L, Brault V, Suter U, Taketo MM, Kemler R, Sommer L (2004) Instructive role of Wnt/beta-catenin in sensory fate specification in neural crest stem cells. Science 303:1020-1023.

Lee SM, Tole S, Grove E, McMahon AP (2000) A local Wnt-3a signal is required for development of the mammalian hippocampus. Development 127:457-467.

Lewis JL, Bonner J, Modrell M, Ragland JW, Moon RT, Dorsky RI, Raible DW (2004) Reiterated Wnt signaling during zebrafish neural crest development. Development 131:1299-1308. 
Maretto S, Cordenonsi M, Dupont S, Braghetta P, Broccoli V, Hassan AB, Volpin D, Bressan GM, Piccolo S (2003) Mapping Wnt/beta-catenin signaling during mouse development and in colorectal tumors. Proc Natl Acad Sci USA 100:3299-3304.

Masai I, Heisenberg CP, Barth KA, Macdonald R, Adamek S, Wilson SW (1997) Floating head and masterblind regulate neuronal patterning in the roof of the forebrain. Neuron 18:43-57.

Miyashita-Lin EM, Hevner R, Wassarman KM, Martinez S, Rubenstein JL (1999) Early neocortical regionalization in the absence of thalamic innervation. Science 285:906-909.

Muroyama Y, Fujihara M, Ikeya M, Kondoh H, Takada S (2002) Wnt signaling plays an essential role in neuronal specification of the dorsal spinal cord. Genes Dev 16:548-553.

Nakagawa Y, O’Leary DDM (2002) Patterned expression of orphan nuclear receptor genes ROR- $\alpha$ and $-\beta$ in developing mouse forebrain. Soc Neurosci Abstr 28:626.14.

Nordstrom U, Jessell TM, Edlund T (2002) Progressive induction of caudal neural character by graded Wnt signaling. Nat Neurosci 5:525-532.

Pinson KI, Brennan J, Monkley S, Avery BJ, Skarnes WC (2000) An LDLreceptor-related protein mediates Wnt signalling in mice. Nature 407:535-538.
Rubenstein JL, Martinez S, Shimamura K, Puelles L (1994) The embryonic vertebrate forebrain: the prosomeric model. Science 266:578-580.

Rubenstein JL, Anderson S, Shi L, Miyashita-Lin E, Bulfone A, Hevner R (1999) Genetic control of cortical regionalization and connectivity. Cereb Cortex 9:524-532.

Steinmayr M, Andre E, Conquet F, Rondi-Reig L, Delhaye-Bouchaud N, Auclair N, Daniel H, Crepel F, Mariani J, Sotelo C, Becker-Andre M (1998) Staggerer phenotype in retinoid-related orphan receptor alphadeficient mice. Proc Natl Acad Sci USA 95:3960-3965.

Tamai K, Semenov M, Kato Y, Spokony R, Liu C, Katsuyama Y, Hess F, Saint-Jeannet JP, He X (2000) LDL-receptor-related proteins in Wnt signal transduction. Nature 407:530-535.

Wehrli M, Dougan ST, Caldwell K, O’Keefe L, Schwartz S, Vaizel-Ohayon D, Schejter E, Tomlinson A, DiNardo S (2000) Arrow encodes an LDLreceptor-related protein essential for Wingless signalling. Nature 407:527-530.

Zeltser LM, Larsen CW, Lumsden A (2001) A new developmental compartment in the forebrain regulated by Lunatic fringe. Nat Neurosci 4:683-684.

Zhou CJ, Zhao C, Pleasure SJ (2004) Wnt signaling mutants have decreased dentate granule cell production and radial glial scaffolding abnormalities. J Neurosci 24:121-126. 